Ann. Biol. anim. Bioch. Biophys., I974, 14 (3), 4I7-426.

\title{
HISTOENZYMOLOGIE DE L'OVAIRE CHEZ LA TAUPE. ÉVOLUTION DES POTENTIALITÉS STÉROÏDOGÈNES DE L'INTERSTITIELLE MÉDULLAIRE AU COURS DU CYCLE SEXUEL ANNUEL
}

\author{
A. GOUGEON (1) \\ Laboratoive de Physiologie comparée, \\ Faculté des Sciences, \\ 86022 Poitiers
}

RÉSUMÉ

Chez la Taupe, les cellules de l'interstitielle ovarienne sont soumises à des variations morphologiques cycliques.

De forte taille pendant l'œstrus et le début de l'anœstrus estival, elles sont le siège d'activités $\triangle 5-3 \beta$ - et $20 \alpha$-HSD très importantes, suggérant une libération de $20 \alpha$-dihydroprogestérone au moment de l'ovulation et de l'entrée en repos sexuel.

Pendant la gestation, les cellules interstitielles sont atrophiées et peu actives.

Les amas cellulaires de la médulla ovarienne métaboliquement toujours actifs (succinodeshydrogénase) ne métabolisent pas les stéroïdes.

\section{INTRODUCTION}

L’appareil génital de la Taupe femelle présente des particularités anatomiques intéressantes:

- l'ovaire comporte un cortex renfermant toutes les classes de follicules. En forme de croissant, il est nettement séparé de la médulla composée d'une glande interstitielle massive englobant des " cordons médullaires";

- cet animal à clitoris péniforme est, en outre, doté de glandes annexes de type mâle : prostate et glandes de Cowper.

(1) Nouvelle adresse : Gynécologie Obstétrique, Maternité Hôpital Antoine Béclère, I57, rue de la Porte de Trivaux, 92 I 40 Clamart. 
Nombre d'auteurs ont été attirés par cet aspect original. On peut citer les principaux : Macleod (I880), Limon (I9OI), Tourneux (I904), Aime (I907), Popoff (I9II), Harrison-MatThews (I935), Godet (I949), Huddleston (I964), DeaNESL, (I966).

La plupart d'entre-eux ont assimilé cet ovaire à un ovotestis et émis l'hypothèse que la glande interstitielle sécrèterait des androgènes responsables de la masculinisation du tractus génital.

Nous avons tenté au cours de cette analyse :

- de préciser l'orientation générale du métabolisme stéroïdogène de l'interstitielle ovarienne en étudiant les principales hydroxystéroïde-déshydrogénases (HSD) ;

- de mettre en parallèle pour cette glande interstitielle les variations morphologiques et les variations du niveau d'activité des HSD pendant les différentes phases du cycle sexuel annuel.

\section{MATÉRIEL ET MÉTHODES}

\section{Animaux}

47 taupes femelles capturées dans le département des Deux-Sèvres ont été étudiées. Les animaux sont sacrifiés par décapitation; un ovaire et le reste du tractus génital sont fixés par le Bouin Hollande, l'autre gonade est congelée pour l'analyse histoenzymologique.

\section{Analyse histologique}

Après inclusion à la paraffine, les ovaires sont coupés à $5 \mu$, l'utérus, le vagin et le clitoris à Io $\mu$. Les coupes sont colorées à l'hémalun-picro-indigo-carmin.

\section{Analyse histoenzymologique}

Afin de préciser l'orientation métabolique des clifférents tissus ovariens, nous avons étudié quelques activités enzymatiques pendant les différentes phases du cycle sexuel annuel. La technique utilisée, dérivant de celles de Nachlas et al. (1957) pour la succino-oxydase, et de WatTenBERG (1958) modifiée par LÉvy et al. (1959) pour les HSD, ainsi que les substrats utilisés ont été décrits dans une note précédente (GougEon, 1974).

\section{RÉSULTATS}

La Taupe est un animal monoestrien. Les différentes phases du cycle sexuel annuel sont les suivantes: un proestrus (janvier), un œstrus (janvier à février), une période de gestation (février à mai) puis la lactation (mars à juin) et un anœestrus de 9 mois (avril-mai à janvier).

En raison des modifications de l'histologie ovarienne observées pendant l'été et l'hiver, nous avons divisé l'anostrus en deux parties:

- un anœstrus estival caractérisé par l'existence d'une lutéinisation thécale généralisée et par la présence de cellules interstitielles de taille moyenne à cytoplasme éosinophile ; 
- un anœstrus hivernal où prédominent des follicules primordiaux et jeunes; les cellules interstitielles sont hypertrophiées, leur cytoplasme est faiblement éosinophile.

\section{A. - Évolution histologique de la médullaire ovarienne en fonction du cycle sexuel annuel}

Elle renferme essentiellement :

- des cellules interstitielles du type de celles décrites par BourN (I909);

- des amas cellulaires dont l'aspect varie au cours du cycle sexuel. Baptisés " cordons médullaires" par les auteurs anciens, il ne s'agit pas de cordons proprement. dit comme c'est le cas des tubules séminifères du testicule ou des cordons médullaires de l'ovaire du Desman observés par PEYRE (I96I).

La conformation sphérique de ces amas est démontrée par l'observation en coupe sériée et par l'absence de toute image tubulaire. L'examen en coupe semi fine ne permet pas de confirmer l'hypothèse de GoDET (I949) sur la nature syncitiale de ces formations.

I. Évolution des cellules interstitielles.

L'évolution en fonction du cycle sexuel annuel, de l'aspect histologique de ces cellules est résumée sur le tableau I (fig. 2, 3, 4, Pl. I).

- Au moment de l'œestrus, les cellules interstitielles s'atrophient très vite. Ce phénomène est sans doute imputable à la mobilisation de précurseurs stockés pendant l'anœstrus hivernal, suivie d'une libération de produits de sécrétion.

TABLEAU I

Evolution histologique des portions corticale et médullaire de l'ovaire chez la Taupe, au cours du cycle sexuel annuel

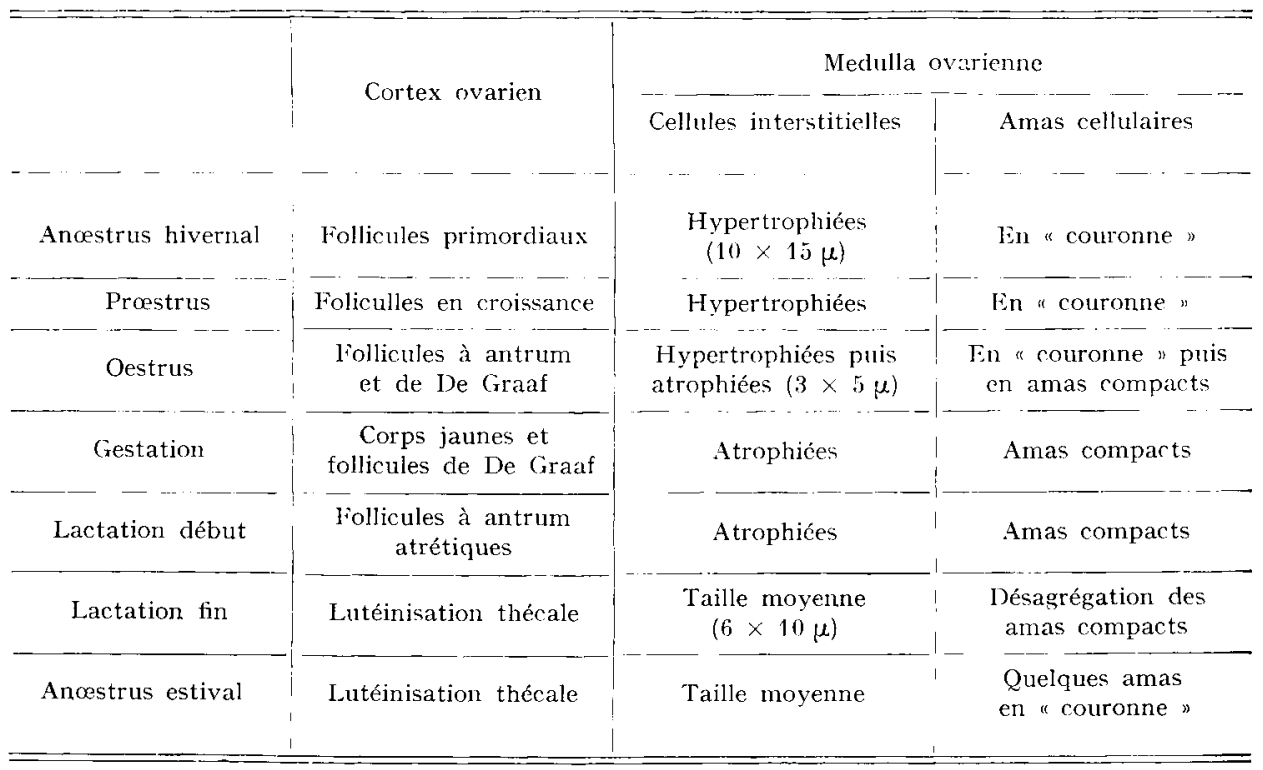


- Lors de l'entrée en anœstrus estival, après la lactation, le volume du tissu interstitiel s'accroît par augmentation de la taille des cellules et par adjonction de nouveaux éléments cellulaires issus de la lutéinisation des thèques de la plupart des follicules (fig. 6, P1. I). Certaines cellules des amas médullaires et certaines cellules conjonctives subiraient la même évolution (POPOFF, IgII ; GODET, I949).

2. Évolution des amas médullaires.

Cette évolution est consignée dans le tableau I.

Nous avons distingué les amas "en couronne ", constitués de cellules riches en cytoplasme peu éosinophile, à noyau rond excentré (fig. 2, P1. I), des ensembles cellulaires compacts pauvres en cytoplasme (fig. 3, P1. I).

Entre ces deux aspects caractéristiques, parfois observés au même moment, il existe de nombreux stades intermédiaires confirmant l'évolution de ces amas d'une forme à l'autre.

\section{B. - Histoenzymologie de l'ovaire pendant le cycle sexuel annuel}

Nous avons étudié l'activité enzymologique des ovaires de 35 Taupes.

La succino oxydase et onze HSD ont été recherchées en présence de 2 I substrats. Les résultats sont consignés sur le tableau 2.

Activité des hydroxystéroïde-déshydrogénases (HSD) et de la succ - : activité nulle; $(+)$ : très faible; + : faib

\begin{tabular}{|c|c|c|c|c|c|c|c|c|c|}
\hline \multirow{2}{*}{$=-1$} & \multirow[b]{2}{*}{$\begin{array}{l}\text { Succinate } \\
\text { de } \mathrm{Na}\end{array}$} & \multicolumn{2}{|c|}{$3 \alpha-\mathrm{HSL}$} & \multicolumn{4}{|c|}{$\Delta_{5}-3 \beta-$ HSD } & \multirow{2}{*}{$\frac{6 \beta-\mathrm{HSD}}{\frac{6 \beta-\mathrm{OH}-}{\mathrm{Pg}}}$} & \multirow{2}{*}{ 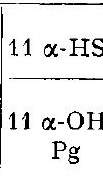 } \\
\hline & & $\begin{array}{l}\text { Andro- } \\
\text { stérone }\end{array}$ & $\begin{array}{l}\text { Etiocho- } \\
\text { lanolone }\end{array}$ & Pn & $\begin{array}{c}17 \alpha-\mathrm{OH}- \\
\operatorname{Pr}\end{array}$ & DHEA & $\begin{array}{l}\text { Andro- } \\
\text { stène- } \\
\text { diol }\end{array}$ & & \\
\hline $\begin{array}{l}\text { Anœestrus } \\
\text { hivernal }\end{array}$ & ++ & - & ++ & ++ & + & ++ & ++ & & - \\
\hline Proestrus & ++ & - & ++ & $+t$ & + & +++ & ++ & & - \\
\hline Oistrus & +++ & - & ++ & +4 & $\frac{1}{+}$ & $+t+$ & $+t+$ & + & - \\
\hline Gestation & +++ & - & ++ & +-+ & + & ++ & ++ & $\overline{+}$ & - \\
\hline $\begin{array}{l}\text { Début de } \\
\text { Iactation }\end{array}$ & $+-t$ & $(+)$ & ++ & +++ & + & +++ & +++ & + & 一 \\
\hline $\begin{array}{l}\text { Fin de la } \\
\text { lactation }\end{array}$ & $+t+$ & $t^{-}$ & ++ & +++ & + & $+t+t$ & $t+t$ & + & - \\
\hline $\begin{array}{l}\text { Anœestrus } \\
\text { estival }\end{array}$ & +++ & + & ++ & $+t+$ & + & $+\frac{1}{1}++$ & +++ & + & - \\
\hline $\begin{array}{l}\text { Coupes te } \\
\text { DH }\end{array}$ & $\begin{array}{l}\text { oins incubé } \\
\text { : Pregnen } \\
\text { : Dehydro } \\
\text { : Progesté }\end{array}$ & $\begin{array}{l}\text { s sans sub } \\
\text { lone; } \\
\text { piandrost } \\
\text { one; }\end{array}$ & $\begin{array}{l}\text { strat } \\
\text { érone; }\end{array}$ & $\begin{array}{l}\mathrm{E}_{1} \\
\mathrm{E}_{2} \\
\mathrm{E}_{3}\end{array}$ & $\begin{array}{l}\text { Tistrone } \\
: \text { Tistradiol } \\
\text { : Gistriol. }\end{array}$ & & & & \\
\hline
\end{tabular}




\section{La succino-déshydrogénase.}

Les grains de diformazan sont uniformément répartis dans la médulla. Les cellules interstitielles et les îlots médullaires montrent une activité métabolique d'intensité moyenne sensiblement égale toute l'année (fig. I, Pl. II).

Au niveau des follicules, la thèque interne est plus riche en cette enzyme que la granulosa.

C'est le corps jaune qui présente, pendant la gestation, la plus forte activité succino-déshydrogénase (fig. 2, P1. III).

\section{Les hydroxystéroide déshydrogénases.}

Certaines enzymes : $3 \alpha-, \Delta_{5}-3 \beta, 6 \beta-$, I7 $\beta$ - et $20 \alpha$-HSD ont été mises en évidence ; d'autres : II $\alpha$-, II $\beta$-, I $6 \alpha$, I6 $\beta$ - et $20 \beta$-HSD sont toujours absentes.

- Aucune HSD n'est mise en évidence dans les cellules des amas médullaires, quel que soit leur état (fig. 2, 3, 4, P1. II).

- Les cellules interstitielles sont également riches en $\Delta_{5}-3 \beta$ - et en $20 \alpha$-HSD, l'intensité du dépôt de diformazan variant avec la phase du cycle sexuel annuel.

Le maximum d'activité $\left(\Delta_{5}-3 \beta\right.$ et $20 \alpha$-HSD) est observé pendant l'œstrus (lorsque les cellules interstitielles sont encore hypertrophiées) et le début de l'anœstrus estival ; le minimum intervient pendant la gestation (fig. 2, 3, 4, P1. II).

dase au niveau de l'insterstitielle ovarienne chez la Taupe

+ : moyenne; +++ : forte; ++++ : très forte

\begin{tabular}{|c|c|c|c|c|c|c|c|c|c|c|c|}
\hline \multicolumn{2}{|c|}{$11 \beta-H S D$} & \multicolumn{2}{|c|}{$16 \alpha-\mathrm{HSD}$} & \multicolumn{2}{|c|}{$16 \beta-\mathrm{HSD}$} & \multirow{2}{*}{$\frac{17 \alpha-\operatorname{HSD}}{17 \alpha-\mathrm{E}_{2}}$} & \multicolumn{2}{|c|}{$17 \beta$-HSD } & \multicolumn{2}{|c|}{$20 \alpha-H S D$} & \multirow{2}{*}{$\begin{array}{c}20 \beta-H S D \\
20 \beta-O H- \\
\text { Pg }\end{array}$} \\
\hline $\begin{array}{l}11 \beta- \\
\text { indro- } \\
\text { snediol }\end{array}$ & Cortisol & $\begin{array}{c}16 \alpha \\
\mathrm{OH}-\mathrm{E}_{1}\end{array}$ & $E_{3}$ & $\begin{array}{c}16 \beta- \\
\mathrm{OH} \mathrm{E}_{1}\end{array}$ & Epi $\mathrm{E}_{3}$ & & $\begin{array}{l}\text { Testo- } \\
\text { stérone }\end{array}$ & $\begin{array}{c}17 \beta- \\
\mathrm{E}_{2}\end{array}$ & $\begin{array}{c}20 \alpha-\mathrm{OH} \\
\mathrm{Pg}\end{array}$ & $\begin{array}{l}\text { Pregna- } \\
\text { nediol }\end{array}$ & \\
\hline 一 & - & - & - & - & - & - & $(+)$ & $(+)$ & $+t$ & $(+)$ & 一 \\
\hline- & - & - & - & - & - & - & $(+)$ & $(+)$ & ++ & $(+)$ & - \\
\hline - & - & - & - & - & - & - & $(+)$ & - & $+++-t$ & $(+)$ & - \\
\hline - & - & - & - & - & - & - & $(+)$ & - & ++ & $(+)$ & - \\
\hline - & - & 一 & - & - & - & - & $(+)$ & - & +++ & $(+)$ & - \\
\hline - & 一 & - & 一 & - & - & $(+)$ & + & t. & $+++t$ & $(+)$ & - \\
\hline$\ldots$ & - & 一 & - & - & - & $(+)$ & + & $(+)$ & $t+t+$ & $(+)$ & - \\
\hline
\end{tabular}


- Les cellules de la granulosa et les thèques internes présentent une faible activité $\Delta_{5}-3 \beta$-HSD pendant le proestrus et l'œstrus. Pendant la gravidité, les thèques internes et les corps jaunes, très riches en cette enzyme, sont alors les seuls tissus ovariens à haut niveau d'activité stéroïdogène (fig. $3, \mathrm{Pl}$. III).

- Les $3 \alpha-, 6 \beta$ - et I7 $\beta$-HSD peuvent être mises en évidence tout au long de l'année dans la glande interstitielle, les thèques internes et les corps jaunes.

- Les $6 \beta$ - et I7 $\beta$-HSD le sont dans les cellules de la granulosa. Cependant en dehors de la $3 \alpha$-HSD (fig. I, P1. III) toutes ces enzymes sont faiblement actives.

\section{DISCUSSION}

Chez la Taupe, l'évolution de la glande interstitielle au cours du cycle sexuel annuel est très originale, comparée à cette même évolution chez d'autres mammifères.

Pendant la gestation, contrairement à ce que l'on observe chez la Lapine (REGaud, I908), le Myotis (Herlant, I956), l'Écureuil (Sfrth et Prasad, I967), les rongeurs (Mossmax, I964), la Femme (Guraya, I972) la glande interstitielle est atrophiée et peu active. Les $\Delta_{5}-3 \beta$ - et $20 \alpha$-HSD, bien mises en évidence pendant l'œstrus, sont alors plus difficilement révélées.

Cependant, certaines espèces, telles la Chatte (Julliard et Malassine, I973) et l'Hermine (Gulamhusein et Thawley, I972), montrent une évolution analogue à celle de la Taupe.

Chez d'autres animaux monostriens (SETH et PRASAD, I967) ou polyœstriens (PEYRE, I96I) l'anœistrus est caractérisé par une atrophie de l'interstitielle. Chez la Taupe, au contraire, elle est floride et stéroïdogéniquement active, surtout après la lactation.

Par contre la similitude avec les autres espèces est frappante au moment de 1 'oestrus. On observe une chute brutale du volume cellulaire ; il y aurait au moment de l'ovulation, libśration rapide d'un stérö̈de, synthétisó à partir de précurseurs stockés dans les cellules interstielles pendant l'anoestrus.

L'histoenzymologie montre que la glande interstitielle, de même que les autres tissus ovariens (thèque interne, granulosa et corps jaune) métabolise des stéroides.

Si ces derniers présentent tous une activité $\Delta_{5}-3 \beta-H S D$ plus ou moins intense, seule la thèque interne est parfois, comma le tissu interstitiel, le siège d'une activité $20 \alpha$-HSD (fig. 4, Pl. III).

Cette mise en évidence de la $20 \alpha$-HSD au niveau de la thèque interne des follicules peut paraittre contradictoire avec l'existence d'une synthèse d'œstrogènes habituellement invoquée pour ce tissu. Cependant, cette enzyme est surtout observée après la gestation. A ce moment, la plupart des follicules sont atrétiques. Sans doute intervient-il une "levée d'inhibition " aboutissant à une orientation différente du métabolisme des cellules thécales. Celles-ci possèdent alors les mêmes enzymes de la stéroïdogenèse que les cellules de la glande interstitielle à la croissance de laquelle elles contribueront ultérieurement.

Que signifie la présence de la $20 \alpha$-HSD?

$\mathrm{Au}$ moment de l'ovulation, il y aurait synthèse et libération de $20 \alpha-\mathrm{OH}-\mathrm{Pg}$, 
cette décharge n'est pas sans rappeler ce que l'on observe chez la Lapine, autre mammifère à ovulation provoquée.

A l'entrée en ancstrus estival, il y aurait une nouvelle synthèse de $20 \alpha-\mathrm{OH}-\mathrm{Pg}$, dont le rôle reste inconnu.

Les résultats obtenus avec les autres enzymes sont difficiles à interpréter, en particulier la présence d'une $3 \alpha$-HSD au sein d'un tissu qui sécrète surtout des progestagènes.

Pourtant cette enzyme a souvent été mise en évidence par des techniques histochimiques au niveau du corps jaune dont on sait qu'il synthétise surtout de la progestérone (Goldman et al., I965, Rat ; BaIliıe et al., I966; HART et al., Ig66, Souris).

Chez la Taupe, Goder (I949) attribue une sécrétion d'androgènes à 1'interstitielle médullaire; l'interstitielle d'origine thécale libérant quant à elle des ostrogènes.

- L'existence de 2 types d'interstitielles est infirmée par l'analyse histoenzymologique. Toute la glande interstitielle réagit de façon homogène quelle qu'en soit l'histogenèse.

- L'idée d'une libération d'androgènes était suggérée par le clivage du repli balano préputial du clitoris (considéré comme un excellent indice de masculinisation) contemporain de l'hypertrophie des cellules interstitielles ovariennes après administration de gonadotropine sérique. Cette affirmation étant cependant nuancée par l'absence de réactivité de l'épithélium prostatique des Taupes femelles traitées.

On peut se demander à la lumière des résultats obtenus au cours de cette étude quelle est 1'hormone responsable de ce clivage?

Chez la Taupe, l'ouverture du repli-balano-préputial correspond en règle générale au moment où l'activité $20 \alpha$-HSD est la plus forte, particulièrement au début de 1'anœstrus estival.

STEINACH et KUN (I93I) ont induit le clivage du repli-balano-préputial clitoridien chez le cobaye en injectant des extraits de corps jaune de Truie.

Or Cook et al. (I967) ont montré que chez la Truie, le corps jaune produit, outre la progestérone, la $\mathrm{I} 7 \alpha$-OH-Progestérone et la 4 androstènedione, de la $20 \alpha$-dihydroprogestérone.

Dans ce second cas, la $20 \alpha$-dihydroprogestérone pourrait être elle aussi impliquée dans un processus de masculinisation, bien que, l'activité des autres hormones ne puisse être, a priori, écartée.

En conclusion, on peut penser que la glande interstitielle de l'ovaire chez la Taupe sécrète de la $20 \alpha$-dihydroprogestérone. Cependant, seule une étude biochimique ultérieure permettrait de confirmer cette hypothèse.

Rę̧u pour publication en janvier 1974.

\section{REMERCIEMENTS}

Nous tenons à remercier M. Pevet ainsi que MM. les Professeurs Peyre et Thibault de l'hospitalité et des facilités qu'ils nous ont accordées pour la réalisation de ce travail. 


\title{
SUMMARY
}

HISTOENZYMOLOGY OF THE OVARY IN THE MOLE. EVOLUTION OF THE STEROIDOGENIC POTENTIALITIES OF THE MEDULLARY INTERSTITIAL,

\author{
DURING THE ANNUAI, SEX CYCLE
}

In the mole, the the ovarian interstitial cells undergo cyclic morphological variation.

Large during oestrus and at the beginning of summer anoestrus, they are the seat of very great $\triangle_{5}-3 \beta$-and $20 \alpha$-HSD activities, thus suggesting $20 \alpha$-dihydroprogesterone surge at the time of ovulation and the beginning of sexual rest.

Interstitial cells are atrophied and not very active during gestation.

The cell masses of the ovarian medulla, always letabolically active (succino dehydrogenase), do not metabolize steroids.

\section{RÉFÉRENCES BIBLIOGRAPHIQUES}

Arme P., I907. Recherches sur les cellules interstitielles de l'ovaire de quelques mammifères. Archs Zool. expér. gén., $4^{\mathrm{e}}$ série, 95-I43.

Baillie A. H., Calman K. C., Ferguson M. M., Hart D. Mck, ig66. Histochemical utilization of $3 \alpha$-, $6 \beta$-, I I $\alpha$-, I $2 \alpha$; I $6 \alpha$-, I7 $\alpha$-, 2 I- et 24 -Hydroxysteroids. $J$. Endocr., 34, I-I 2 .

Bouin P., Ancel P., rgog. Sur les homologies et la signification des glandes à sécrétion interne de l'ovaire. C. R. Soc. Biol., 67, 464 .

Cook B., Kaltenbach C. C., Norton H. W., Nalbandov A. V., i967. Synthesis of progesterone in vitro by porcine corpora lutea. Endocrinology, 81, 573-584.

Deanesly R., I966. Observations on the reproduction in the Mole, Talpa europea. In Rowlands I. W., comparative biology of reproduction in mammals, 387-401, Academic Press, London and New York.

Godet R., I949. Recherches d'anatomie, d'embryologie normale et expérimentale sur l'appareil génital de la Taupe. Bull. Biol. Fr. Belg., 83, 25-III.

Goldman A. S., Yacovac W. C., Bongiovani A. M., 1965. Persistent effets of a synthetic androstene derivative on activities of 3 B-Hydroxy steroid deshydrogenase and glucose- 6 -phosphate deshydrogenase in rats. Endocrinology, 7\%, I I05-II 18.

GougEon A., I974. Aspects originaux de la glande interstitielle ovarienne chez le Hérisson (Morphologie, histogenèse hyperplasie, enzymes de la stérö̈dogenèse. Ann. Biol. Anim. Biochim. Biophys., 14 (sous presse).

Gulamhusein A. P., Thawley A. R., I972. Ovarian cycle and plasma progesterone levels in the female stoat mustela erminea. J. Report. Fert., 31, 492-493.

GURAYA S. S., I972. Function of the human ovary during pregnancy as revealed by histochemical, biochemical and electron microscope techniques, Acta Endocr., 69, ro7-II8.

Harrison-Matthews L., i935. The estrous cycle and intersexuality in the female Mole. Proc. Zool. Soc. London, 1, 347-383.

Hart D. Mck., Baillie A. H., Calman K. C., Ferguson M. M., ig66. Hydroxysteroid development in the mouse adrenal and gonads. J. Anat., 100, 80I-8I2.

Herlant M., I956. Corrélations hypophyso génitales chez la femelle de la Chauve-souris, Myotis myotis (Borkhausen). Archs Biol., 67, 89-180.

Huddeeston T., I965. Some aspects of the cyclic changes in the ovary of the Mole (Talpa europea). M. Sc. Thesis, Bangor, $3 \mathrm{I}$ p.

Juillard M. T., Malassine A., I974. Histoenzymologie de l'ovaire de Chatte non gestante et gestante, importance du tissu interstitiel. C. R. Soc. Biol. (sous presse).

Lévy H., Deane H. W., Rubin B.-L., 1959. Visualisation of steroid-3 $\beta$-ol-dehydrogenase activity in tissue of intact and hypophysectomized Rats. Endocrinology, 65, 932-942.

Limon M., I902-I903. Étude histologique et histogénique de la glande interstitielle de l'ovaire. Archs Anat. micr. Morphol. expér., 5, 155-188.

MAC LEOD J., I88o. Contribution à l'étude de la structure de l'ovaire des mammifères. Archs Biol., 1, 24 I. 
Mossman H. W., 1966. The rodent ovary. In Rowlands I. W., Comparative biology of reproduction in mammals, 455-47o. Academic Press, London and New York.

Nachlas M.-M., Tsou K.-C., Desouza E., Cheng S.-S., Seligman A. M., I957. Cytochemical demonstration of succinic dehydrogenase by the use of a new $p$-nitro phenyl substitued ditetrazole salt. J. Histochem. Cytochem, 5, 420-436.

PEYRE A., I961. Recherche sur l'intersexualité spécifique chez Galemys pyrenaicus, mammifère insectivore. Thèse Doctorat es Sciences Naturelles, Toulouse, $n^{\circ}$ C. N. R. S., I85, I74 pages.

Popoff N., r9i r. Le tissu interstitiel et les corps jaunes de l'ovaire. Archs Biol., 26, 483-459.

Regaud C., Igo8. Gravidité et glande interstitielle de l'ovaire chez la Lapine. C. R. Soc. Biol., $64,396$.

SETh P., Prasad M.- R.-N., I967. Seasonal changes in the histochemical localization of $\Delta_{5}-3 \beta-H y d r o-$ xysteroid dehydrogenase in the ovary of the five-striped Indian palm squirel Funambulus pennanti (Wroughton). Gen. Comp. Endocr., 9, 383-39o.

Steinach, Kuhn. Cités par Goder, 1949.

Tourneux F., Ig04. Hermaphrodisme de la glande génitale chez la Taupe adulte et localisation des cellules interstitielles dans le segment spermatique. Bibl. Anat., 62.

WAtTENBERG L.-W., I958. Microscopic histochemical demonstration of Steroid $3 \beta$-ol-dehydrogenase in tissue sections. J. Histochem. Cytochem., 6, 225-232. 


\section{PLANCHE I}

FIG. I

Ovaire de Taupe: le cortex, en forme de croissant, renferme toutes les classes de follicules. Noter l'importance de la portion médullaire (méd.) ( $X$ Io).

FIG. 2

Estrus: les cellules interstitielles (c. i.) sont hypertrophiées. Les cellules des amas médullaires (a.m.) sont disposées en couronne ( $X$ I 000).

FIG. 3

Gestation: Les cellules interstitielles (c.i.) sont atrophiées, les amas médullaires (a.m.) sont compacts ( $X$ I ooo).

FIG. 4

Début de l'ancestrus estival: les cellules interstitielles (c.i.) ont une taille moyenne. Les amas médullaires (a.m.) ont à nouveau leur aspect « en couronne " ( $\times$ I ooo).

FIG. 5

Amas médullaires: les limites cellulaires sont bien visibles. Coupe semi fine colorée au bleu de Toluidine ( $X$ I ooo).

FIG. 6

Début de l'ancestrus estival: lutéinisation des thèques folliculaires au niveau du cortex ( $X$ I60). 

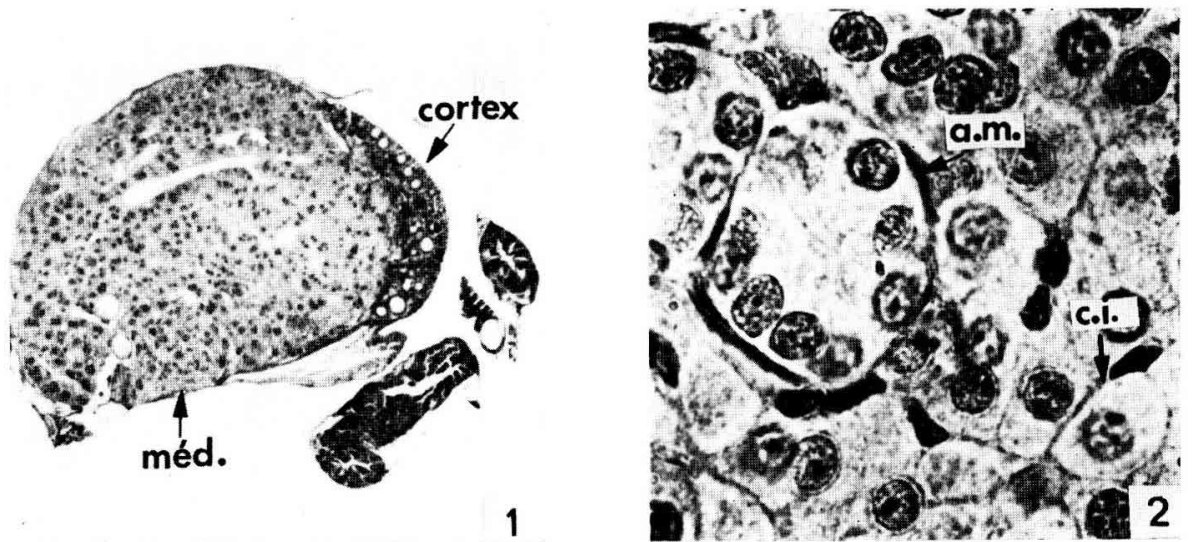

notore

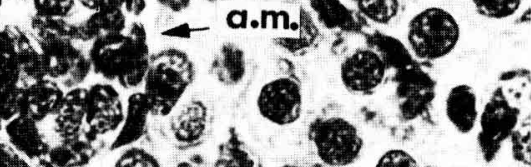

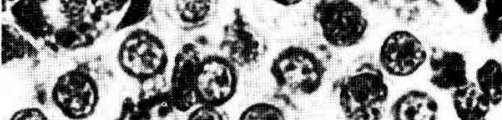

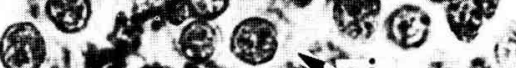

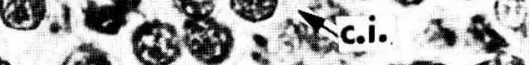
- 1010

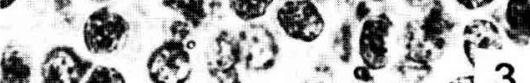
100.23
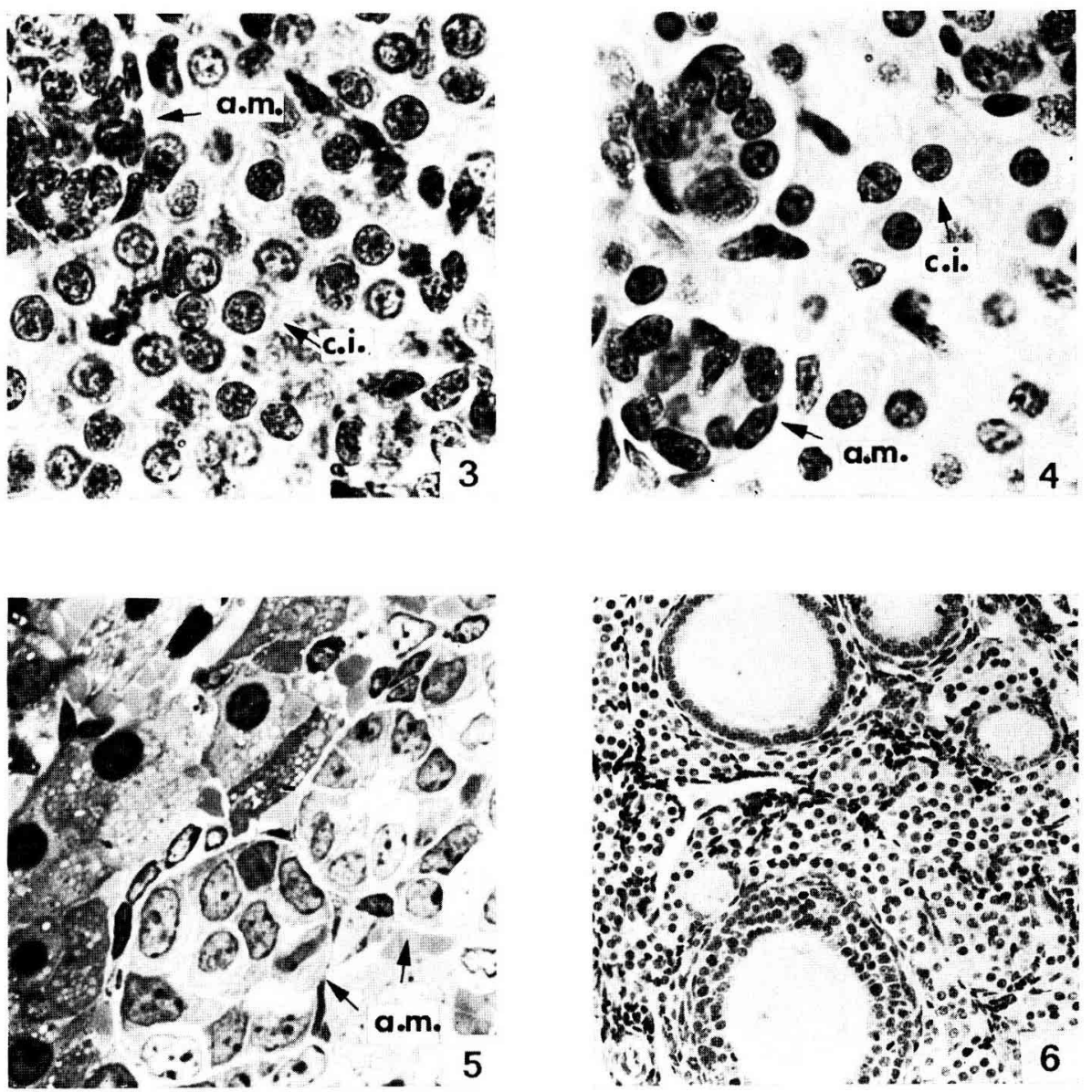


\title{
PLANCHE II
}

\author{
FIG. 1
}

Début de l'anœestrus estival : Succino déshydrogénase. Les cellules interstitielles (c. i.) et les amas médullaires (a. m.) sont le siège d'une activité métabolique importante. $\times 250$.

FIG. 2

Oestrus, $20 \alpha-H$. S. D. : très forte activité des cellules interstitielles (c. i.), pas d'activité au niveau des amas médullaires. $\times 250$.

FIG. 3

Gestation, $20 \alpha-H . S . D$. : Activité moyenne des cellules interstitielles (c. i.), nulle au niveau des amas médullaires (a. m.). $\times 250$.

\section{Fig. 4}

Début de l'ancestrus estival, $20 \alpha-H . S . D$. : très forte activité des cellules interstitielles (c. i.), activité nulle au niveau des amas médullaires (a. m.). $\times 250$. 


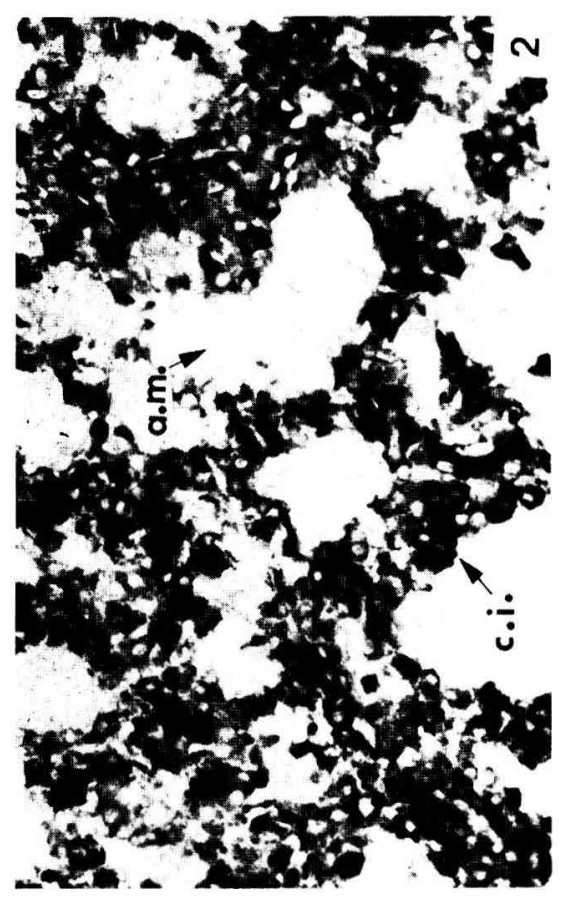

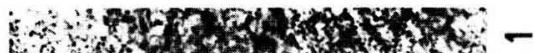

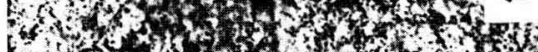

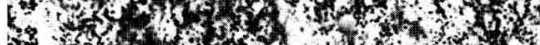

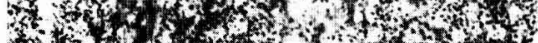

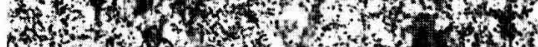
13.

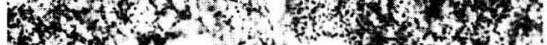

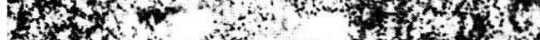

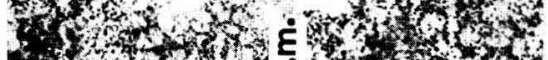

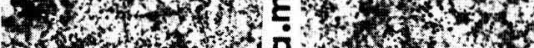

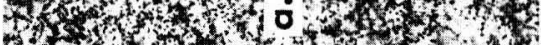

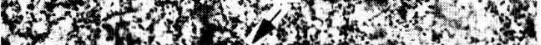

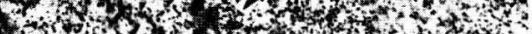
- 1 .

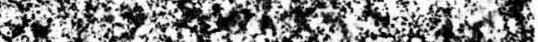

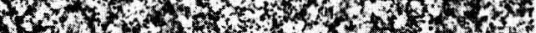

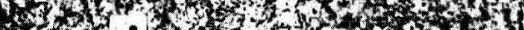

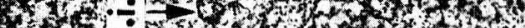

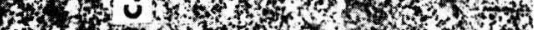

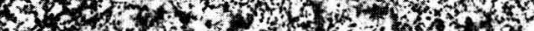

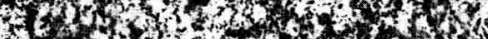

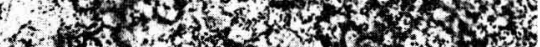

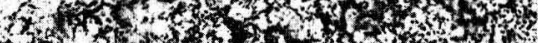

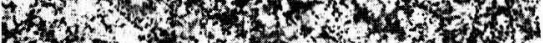

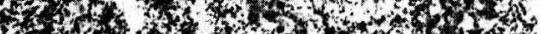

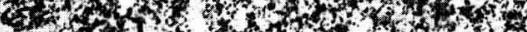

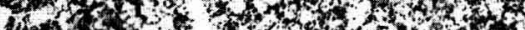
Wow on

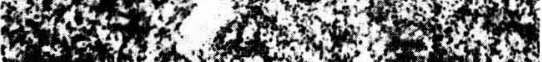

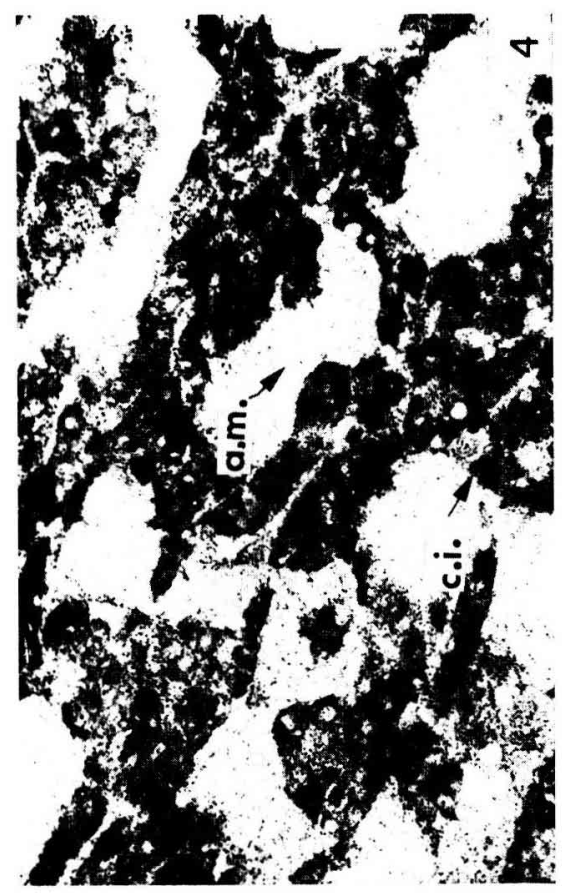

कo

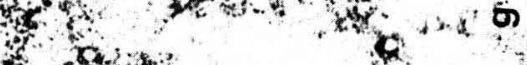

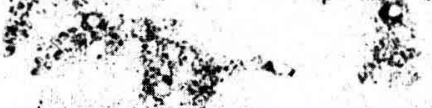

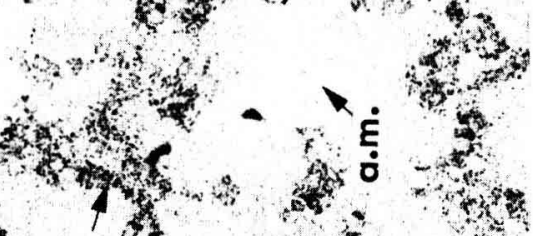

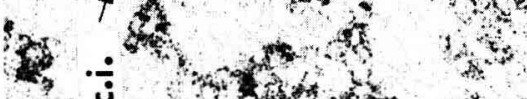

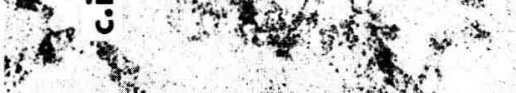

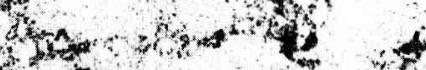

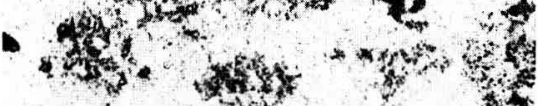

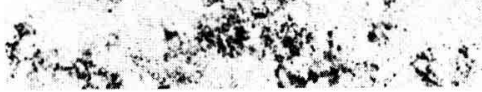


PLANCHE III

FIG. 1

Entrée en anoestrus estival, $3 \propto-H . S . D$. : Activité moyenne des cellules interstitielles (c. i.). $\times 250$.

\section{FIG. 2}

Gestation, succino déshydrogénase : l'activité métabolique du corps jaune (c. j.) est supérieure à celle de la glande interstitielle (g. i.) et à celle du follicule (foll.). $\times 100$.

FIG. 3

Gestation, $\Delta^{5}-3 \beta-H$. S. D. : forte activité du corps jaune (c. j.) et de la thèque interne (t. i.) des follicules. Les cellules de la granulosa (gr.) et les cellules interstitielles (c. i.) présentent une activité moyenne. $\times 100$.

FIG. 4

Lactation, $20 \propto-H . S$. D. : présence d'une activité importante au niveau de la thèque interne d'un follicule atrétique (t. i.), à droite, la glande interstitielle (g. i.) montre aussi une forte activité $20 \propto H . S . D . \times 100$. 

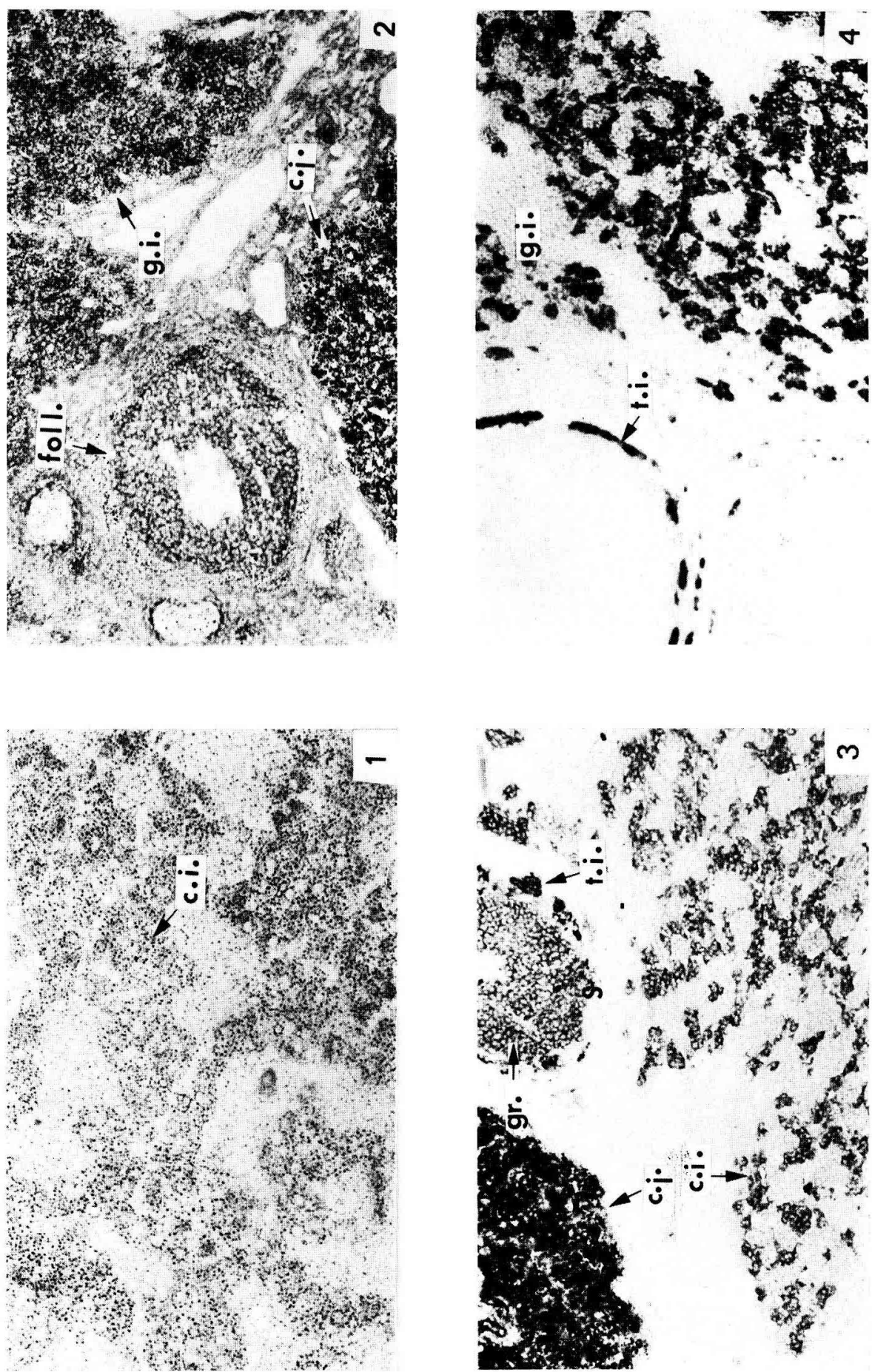

A. GOUGEON 